\title{
Uniqueness of Meromorphic Functions Sharing a Small Func- tion with Their Differential Polynomials
}

\author{
ABHIJIT BANERJEE \\ Department Of Mathematics, West Bengal State University, Barasat, North 24 \\ Prgs., West Bengal, India \\ e-mail : abanerjee_kal@yahoo.co.in
}

ABSTRACT. With the aid of weakly weighted sharing and a recently introduced sharing notion in [3] known as relaxed weighted sharing we investigate the uniqueness of meromorphic functions sharing a small function with its differential polynomials. Our results will improve and supplement all the results obtained by Zhang and Yang [17] as well as a substantial part of the results recently obtained by the present author [2] and thus provide a better answer to the questions posed by $\mathrm{Yu}[14]$ in this regard.

\section{Introduction definitions and results}

Let $f$ and $g$ be two non-constant meromorphic functions defined in the open complex plane $\mathbb{C}$. A meromorphic function $a$ is said to be a small function of $f$ provided that $T(r, a)=S(r, f)$, that is $T(r, a)=o(T(r, f))$ as $r \longrightarrow \infty$, outside of a possible exceptional set of finite linear measure. We denote by $S(f)$ the set of all small functions of $f$. If for some $a \in S(f) \cap S(g), f-a$ and $g-a$ have the same set of zeros with the same multiplicities, we say that $f$ and $g$ share $a$ CM (counting multiplicities), and if we do not consider the multiplicities then $f$ and $g$ are said to share $a$ IM (ignoring multiplicities). We denote by $T(r)$ the maximum of $T(r, f)$ and $T(r, g)$. The notation $S(r)$ denotes any quantity satisfying $S(r)=o(T(r))$ as $r \longrightarrow \infty$, outside of a possible exceptional set of finite linear measure. Let $N_{E}(r, a ; f, g)\left(\bar{N}_{E}(r, a ; f, g)\right)$ be the counting function (reduced counting function) of all common zeros of $f-a$ and $g-a$ with the same multiplicities and $N_{0}(r, a ; f, g)$ $\left(\bar{N}_{0}(r, a ; f, g)\right)$ be the counting function (reduced counting function) of all common zeros of $f-a$ and $g-a$ ignoring multiplicities.

If

$$
\bar{N}(r, a ; f)+\bar{N}(r, a ; g)-2 \bar{N}_{E}(r, a ; f, g)=S(r, f)+S(r, g)
$$

then we say that $f$ and $g$ share $a$ "CM". On the other hand if

$$
\bar{N}(r, a ; f)+\bar{N}(r, a ; g)-2 \bar{N}_{0}(r, a ; f, g)=S(r, f)+S(r, g)
$$

Received September 2, 2008; accepted October 24, 2008.

2000 Mathematics Subject Classification: 30D35.

Key words and phrases: meromorphic function, derivative, small function, weakly weighted sharing. 
then we say that $f$ and $g$ share $a$ "IM". We denote by $L=L(f)$ the following differential polynomial.

$$
L(f)=f^{(k)}+a_{k-1} f^{(k-1)}+\cdots+a_{0} f,
$$

where $a_{j}(j=0,1, \cdots, k-1)$ are small meromorphic functions of $f$.

R. Brück [4] first considered the uniqueness problems of an entire function sharing one value with its derivative and proved the following result.

Theorem $\mathbf{A}([4])$. Let $f$ be a non-constant entire function. If $f$ and $f^{\prime}$ share the value $1 C M$ and if $N\left(r, 0 ; f^{\prime}\right)=S(r, f)$ then $\frac{f^{\prime}-1}{f-1}$ is a nonzero constant.

In the same paper Brück [4] posed the following conjecture.

Conjecture 1.1. Let $f$ be a non-constant entire function, $\rho_{1}(f)$ be the first iterative order of $f$. If $\rho_{1}(f)<\infty$ and $\rho_{1}(f)$ is not a positive integer and if $f$ and $f^{\prime}$ share one finite value $a \mathrm{CM}$ then $\frac{f^{\prime}-a}{f-a}=c$ for some $c \in \mathbb{C} /\{0\}$.

In $2003 \mathrm{Yu}$ [14] investigated the uniqueness problem of an entire or a meromorphic function with its derivative and considered the case when $a$ is a small function. $\mathrm{Yu}$ proved the following two theorems.

Theorem $\mathbf{B}([\mathbf{1 4}])$. Let $f$ be a non-constant entire function, $a \in S(f)$ and $a \not \equiv 0, \infty$. If $f-a$ and $f^{(k)}-a$ share $0 C M$ and $\delta(0 ; f)>\frac{3}{4}$ then $f \equiv f^{(k)}$.

Theorem $\mathbf{C}([\mathbf{1 4}])$. Let $f$ be a non-constant non-entire meromorphic function, $a \in S(f)$ and $a \not \equiv 0, \infty$. If

i) $f$ and a have no common poles,

ii) $f-a$ and $f^{(k)}-a$ share the value $0 C M$,

iii) $4 \delta(0 ; f)+2(8+k) \Theta(\infty ; f)>19+2 k$,

then $f \equiv f^{(k)}$ where $k$ is a positive integer.

In the same paper $\mathrm{Yu}[14]$ posed the following open questions.

(i) Can a CM shared be replaced by an IM shared value in Theorem B ?

(ii) Can the condition $\delta(0 ; f)>\frac{3}{4}$ of Theorem B be further relaxed?

(iii) Can the condition (iii) in Theorem $\mathrm{C}$ be further relaxed ?

(iv) Can in general the condition (i) of Theorem C be dropped? 
Progress to explore the possible answer of $\mathrm{Yu}[14]$ has been remarkable recently, see [1], [9]-[10], [15]-[17]. In 2004, P. Liu and Y. X. Gu [11] provided affirmative answers to the last three questions of $\mathrm{Yu}[14]$. To state the next results we require the following definition known as weighted sharing of values which measure how close a shared value is to be shared IM or to be shared CM.

Definition 1.1([6], [7]). Let $k$ be a nonnegative integer or infinity. For $a \in \mathbb{C} \cup\{\infty\}$ we denote by $E_{k}(a ; f)$ the set of all $a$-points of $f$, where an $a$-point of multiplicity $m$ is counted $m$ times if $m \leq k$ and $k+1$ times if $m>k$. If $E_{k}(a ; f)=E_{k}(a ; g)$, we say that $f, g$ share the value $a$ with weight $k$.

The definition implies that if $f, g$ share a value $a$ with weight $k$ then $z_{0}$ is an $a$-point of $f$ with multiplicity $m(\leq k)$ if and only if it is an $a$-point of $g$ with multiplicity $m(\leq k)$ and $z_{0}$ is an $a$-point of $f$ with multiplicity $m(>k)$ if and only if it is an $a$-point of $g$ with multiplicity $n(>k)$, where $m$ is not necessarily equal to $n$. We write $f, g$ share $(a, k)$ to mean that $f, g$ share the value $a$ with weight $k$. Clearly if $f, g$ share $(a, k)$, then $f, g$ share $(a, p)$ for any integer $p, 0 \leq p<k$. Also we note that $f, g$ share a value $a$ IM or CM if and only if $f, g$ share $(a, 0)$ or $(a, \infty)$ respectively. If $a$ is a small function we define that $f$ and $g$ share $(a, l)$ which means $f$ and $g$ share $a$ with weight $l$ if $f-a$ and $g-a$ share $(0, l)$. Though we use the standard notations and definitions of the value distribution theory available in [5], we explain some definitions and notations which are used in the paper.

Definition 1.2([9]). Let $p$ be a positive integer and $a \in \mathbb{C} \cup\{\infty\}$.

(i) $N(r, a ; f \mid \geq p)(\bar{N}(r, a ; f \mid \geq p))$ denotes the counting function (reduced counting function) of those $a$-points of $f$ whose multiplicities are not less than $p$.

(ii) $N(r, a ; f \mid \leq p)(\bar{N}(r, a ; f \mid \leq p))$ denotes the counting function (reduced counting function) of those $a$-points of $f$ whose multiplicities are not greater than $p$.

Definition 1.3([13]). For $a \in \mathbb{C} \cup\{\infty\}$ and a positive integer $p$ we denote by $N_{p}(r, a ; f)$ the sum $\bar{N}(r, a ; f)+\bar{N}(r, a ; f \mid \geq 2)+\cdots+\bar{N}(r, a ; f \mid \geq p)$. Clearly $N_{1}(r, a ; f)=\bar{N}(r, a ; f)$.

Definition 1.4([15]). For a positive integer $p$ and $a \in \mathbb{C} \cup\{\infty\}$ we put

$$
\delta_{p}(a ; f)=1-\limsup _{r \longrightarrow \infty} \frac{N_{p}(r, a ; f)}{T(r, f)}
$$

Clearly $0 \leq \delta(a ; f) \leq \delta_{p}(a ; f) \leq \delta_{p-1}(a ; f) \leq \cdots \leq \delta_{2}(a ; f) \leq \delta_{1}(a ; f)=\Theta(a ; f)$.

In 2004 Lahiri and Sarkar [15] also gave some affirmative answers to the first three questions imposing some restrictions on the zeros and poles of $a$. But they did not provide any definite answer corresponding to the question (i) of $\mathrm{Yu}$ as mentioned above. Rather they confined their investigations of sharing of small function up to weight 2. In 2005 Q. C. Zhang [15] studied the problem of meromorphic or entire 
function sharing one small function and improved the results of Lahiri and Sarkar [9] and answered all the four open questions of Yu. Zhang proved the following theorems.

Theorem $\mathbf{D}([\mathbf{1 5}])$. Let $f$ be a non-constant meromorphic function and $k(\geq 1)$, $l(\geq 0)$ be integers. Also let $a \equiv a(z) \quad(\equiv 0, \infty)$ be a meromorphic small function. Suppose that $f-a$ and $f^{(k)}-a$ share $(0, l)$. If $l(\geq 2)$ and

$$
(3+k) \Theta(\infty, f)+2 \delta_{2+k}(0 ; f)>4+k
$$

or $l=1$ and

$$
(4+k) \Theta(\infty, f)+3 \delta_{2+k}(0 ; f)>6+k
$$

or $l=0$ and

$$
(6+2 k) \Theta(\infty, f)+5 \delta_{2+k}(0 ; f)>10+2 k
$$

then $f \equiv f^{(k)}$.

In 2006 Lin and Lin [10] introduced the notion of weakly weighted sharing which we shall define next.

Definition 1.5([10]). Let $f, g$ share $a$ "IM" for $a \in S(f) \cap S(g)$ and $k$ be a positive integer or $\infty$.

(i) $\bar{N}^{E}(r, a ; f, g \mid \leq k)$ denotes the reduced counting function of those $a$-points of $f$ whose multiplicities are equal to the corresponding $a$-points of $g$, both of their multiplicities are not greater than $k$.

(ii) $\bar{N}^{0}(r, a ; f, g \mid>k)$ denotes the reduced counting function of those $a$-points of $f$ which are $a$-points of $g$, both of their multiplicities are not less than $k$.

Definition 1.6([10]). For $a \in S(f) \cap S(g)$, if $k$ be a positive integer or $\infty$ and $\bar{N}(r, a ; f \mid \leq k)-\bar{N}^{E}(r, a ; f, g \mid \leq k)=S(r, f), \bar{N}(r, a ; g \mid \leq k)-\bar{N}^{E}(r, a ; f, g \mid \leq k)=$ $S(r, g)$

$$
\begin{aligned}
& \bar{N}(r, a ; f \mid \geq k+1)-\bar{N}^{0}(r, a ; f, g \mid \geq k+1) \\
= & S(r, f), \bar{N}(r, a ; g \mid \geq k+1)-\bar{N}^{0}(r, a ; f, g \mid \geq k+1) \\
= & S(r, g)
\end{aligned}
$$

or if $k=0$ and

$$
\bar{N}(r, a ; f)-\bar{N}_{0}(r, a ; f, g)=S(r, f), \quad \bar{N}(r, a ; g)-\bar{N}_{0}(r, a ; f, g)=S(r, g),
$$

then we say $f, g$ weakly share $a$ with weight $k$. Here we write $f, g$ share " $(a, k)$ " to mean that $f, g$ weakly share $a$ with weight $k$. 
Obviously if $f, g$ share " $(a, k)$ ", then $f, g$ share " $(a, p)$ " for any integer $p$, $0 \leq p<k$. Also we note that $f, g$ share $a$ "IM" or "CM" if and only if $f, g$ share " $(a, 0)$ " or " $(a, \infty)$ " respectively. With the notion of weakly weighted sharing improving the results of $\mathrm{Yu}[14]$ and Liu-Gu [11] Lin and Lin [10] proved the following results.

Theorem $\mathbf{E}([\mathbf{1 0}])$. Let $f$ be a non-constant meromorphic function and $k(\geq 1)$, $l(\geq 0)$ be integers. Also let $a \in S(f)$ and $a \not \equiv 0, \infty$. Suppose that $f-a$ and $f^{(k)}-a$ share " $(0, l)$ ". If

$2 \leq l \leq \infty$ and

$$
4 \Theta(\infty, f)+2 \delta_{2+k}(0 ; f)>5
$$

or $l=1$ and

$$
\frac{9+k}{2} \Theta(\infty, f)+\frac{5}{2} \delta_{2+k}(0 ; f)>6+\frac{k}{2}
$$

or $l=0$ and

$$
(7+2 k) \Theta(\infty, f)+5 \delta_{2+k}(0 ; f)>11+2 k
$$

then $f \equiv f^{(k)}$.

In [2] the present author has improved all the results of Theorem E by relaxing the conditions (1.4)-(1.6) and obtained the following results.

Theorem $\mathbf{F}([2])$. Let $f$ be a non-constant meromorphic function and $k(\geq 1)$, $l(\geq 0)$ be integers $a \in S(f)$ and $a(z) \not \equiv 0, \infty$. Suppose that $f-a$ and $f^{(k)}-a$ share "(0,l)". If

$2 \leq l \leq \infty$ and

$$
3 \Theta(\infty ; f)+\delta_{2+k}(0 ; f)+\delta_{2}(0 ; f)>4
$$

or $l=1$ and

$$
\frac{7+k}{2} \Theta(\infty ; f)+\delta_{2+k}(0 ; f)+\frac{1}{2} \delta_{1+k}(0 ; f)+\delta_{2}(0 ; f)>5+\frac{k}{2}
$$

or $l=0$ and

$$
(6+2 k) \Theta(\infty ; f)+\delta_{2+k}(0 ; f)+\delta_{1+k}(0 ; f)+\delta_{2}(0 ; f)+2 \Theta(0 ; f)>10+2 k
$$

then $f \equiv f^{(k)}$.

Recently Zhang and Yang [17] not only improved the results of Zhang [15] by weakening all the conditions (1.1)-(1.3) but also replaced $f^{(k)}$ by $L(f)$. They have proved the following theorem.

Theorem $\mathbf{G}([\mathbf{1 7}])$. Let $f$ be a non-constant meromorphic function and $k(\geq 1)$, 
$l(\geq 0)$ be integers. Also, let $a \equiv a(z)(\not \equiv 0, \infty)$ be a small meromorphic function. Suppose that $f-a$ and $L(f)-a$ share $(0, l)$. If $2 \leq l \leq \infty$ and

$$
3 \Theta(\infty, f)+\delta_{2+k}(0 ; f)+\delta_{2}(0 ; f)+\delta(a, f)>4
$$

or $l=1$ and

$$
\frac{7+k}{2} \Theta(\infty, f)+\delta_{2+k}(0 ; f)+\frac{1}{2} \delta_{1+k}(0 ; f)+\delta_{2}(0 ; f)+\delta(a, f)>5+\frac{k}{2}
$$

or $l=0$ and

$(1.12)$

$(6+2 k) \Theta(\infty, f)+\delta_{2+k}(0 ; f)+2 \delta_{1+k}(0 ; f)+\delta_{2}(0 ; f)+\Theta(0 ; f)+\delta(a, f)>10+2 k$

then $f \equiv L(f)$.

It is worth mentioning that recently in [1] the present author has also studied the uniqueness of $f$ and $f^{(k)}$ and improved Theorem D partially by replacing the conditions (1.2) and (1.3) by two weaker ones which are different from that of Zhang and Yang [17]. But in the paper we will concentrate our attention to improve Theorems F-G and consequently provide better answers to the questions of Yu than that was given in [17].

We now state the following two theorems.

Theorem 1.1. Let $f$ be a non-constant meromorphic function and $k(\geq 1), l(\geq 0)$ be integers $a \in S(f)$ and $a(z) \not \equiv 0, \infty$. Suppose that $f-a$ and $L(f)-a$ share " $(0, l)$ ". If

$2 \leq l \leq \infty$ and

$$
3 \Theta(\infty ; f)+\delta_{2+k}(0 ; f)+\delta_{2}(0 ; f)+\delta_{3}(a, f)>4
$$

or $l=1$ and

$$
\frac{7+k}{2} \Theta(\infty, f)+\delta_{2+k}(0 ; f)+\frac{1}{2} \delta_{1+k}(0 ; f)+\delta_{2}(0 ; f)+\delta_{3}(a, f)>5+\frac{k}{2}
$$

or $l=0$ and

$(6+2 k) \Theta(\infty ; f)+\delta_{2+k}(0 ; f)+2 \delta_{1+k}(0 ; f)+\delta_{2}(0 ; f)+\Theta(0 ; f)+\delta_{2}(a ; f)>10+2 k$

then $f \equiv L(f)$.

From Theorem 1.1 we immediately have the following corollary.

Corollary 1.1. Let $f$ be a non-constant meromorphic function and $k(\geq 1), l(\geq 0)$ be integers. Also let $a \in S(f)$ and $a \neq \equiv, \infty$. Suppose that $f-a$ and $f^{(k)}-a$ share " $(0, l)$ ". If

$2 \leq l \leq \infty$ and

$$
2 \delta_{2+k}(0 ; f)+3 \Theta(\infty ; f)+\delta_{3}(a ; f)>4
$$


or $l=1$ and

$$
\frac{5}{2} \delta_{2+k}(0 ; f)+\frac{7+k}{2} \Theta(\infty ; f)+\delta_{3}(a ; f)>5+\frac{k}{2}
$$

or $l=0$ and

$$
5 \delta_{2+k}(0 ; f)+(6+2 k) \Theta(\infty ; f)+\delta_{2}(a ; f)>10+2 k
$$

then $f \equiv f^{(k)}$.

Conditions (1.13)-(1.15) weaken all the conditions of Theorem G. On the other hand the conditions (1.13)-(1.14) are weaker than the conditions (1.7)-(1.8) in Theorem F. Clearly conditions (1.16) and (1.18) provides respectively the better answers corresponding to the second and first question of $\mathrm{Yu}$ [14] than that given by Zhang and Yang [17]. Also (1.16) gives the better answers corresponding to the second question of $\mathrm{Yu}[14]$ than that is given by Banerjee [2]. But whether the condition (1.9) can be further relaxed still remains an open problem.

In the next theorem we shall show that if $l \geq k$, the condition (1.13) stated above can further be weakened at the cost of consideration $a(\neq 0, \infty)$ to be a constant.

Theorem 1.2. Let $f$ be a non-constant meromorphic function and $k(\geq 1), l(\geq 1)$ be integers and $a(\neq 0, \infty)$ be a constant. Suppose that $f-a$ and $L(f)-a$ share "( $0, l)$ ". If $l(\geq k)$ and

$$
3 \Theta(\infty ; f)+\delta_{2+k}(0 ; f)+\delta_{2}(0 ; f)+\delta_{2}(a, f)>4
$$

then $L(f)=f$.

Now it is clear from Definition 1.1 and Definition 1.5 that weighted sharing and weakly weighted sharing are respectively scalings between IM, CM and "IM", "CM". Also weakly weighted sharing includes the definition of weighted sharing.

Recently in [3] another sharing notion known as relaxed weighted sharing has been introduced which is also a scaling between "IM" and "CM" but weaker than weakly weighted sharing and hence include the same definition. We first require the following notation.

Definition 1.7([3]). For $a \in S(f) \cap S(g)$ we denote by $\bar{N}(r, a ; f|=p ; g|=q)$ the reduced counting function of common zeros of $f-a$ and $g-a$ with multiplicities $p$ and $q$ respectively.

We are now at a stage to discuss about the definition of relaxed weighted sharing.

Definition 1.8([3]). For $a \in S(f) \cap S(g)$ let $f, g$ share $a$ "IM". Also let $k$ be a positive integer or $\infty$. If

$$
\sum_{\substack{p, q \leq k \\(p \neq q)}} \bar{N}(r, a ; f|=p ; g|=q)=S(r)
$$


then we say $f, g$ share $a$ with weight $k$ in a relaxed manner. Here we write $f, g$ share $(a, k)^{*}$ to mean that $f, g$ share $a$ with weight $k$ in a relaxed manner.

Obviously if $f, g$ share $(a, k)^{*}$, then $f, g$ share $(a, p)^{*}$ for any integer $p, 1 \leq p<k$. Also we note that $f, g$ share " $(a, 0)$ " or " $(a, \infty)$ " if and only if $f, g$ share $(a, 1) *$ or $(a, \infty) *$ respectively.

We note that $f, g$ share " $(a, k)$ " means they share $(a, k)^{*}$ for $k \geq 1$ but not conversely. In particular when $k \geq 2, f, g$ share " $(a, k-1)$ " implies they share $(a, k)^{*}$ but not conversely. Also from the definition of relaxed weighted sharing it is clear that for finite $k f, g$ share $(a, k)^{*}$ actually means they share $a$ "IM" with some restrictions imposed on the common zeros of $f-a$ and $g-a$ up to multiplicity $k$. In particular if $k=2$ the restrictions are minimum. In the next theorem we will show that if in Theorem $1.1 f$ and $L(f)$ share $(a, 2) *$ instead of " $(a, 0)$ " the condition (1.15) can further be weakened.

We further prove the following.

Theorem 1.3. Let $f$ be a non-constant meromorphic function and $k(\geq 1)$ be an integer, $a \in S(f)$ and $a \neq 0, \infty$. Suppose that $f-a$ and $L(f)-a$ share $(0,2)^{*}$. If

$$
(4+k) \Theta(\infty ; f)+\delta_{2+k}(0 ; f)+\delta_{1+k}(0 ; f)+\delta_{2}(0 ; f)+\delta_{3}(a ; f)>6+k
$$

then $f \equiv L(f)$.

We now give some more definitions.

Definition 1.9. Let $k$ be a positive integer and $f, g$ be two non-constant meromorphic functions such that $f$ and $g$ share the value 1 "IM". Let $z_{0}$ be a zero of $f(z)-1$ of multiplicity $p$ and a zero of $g(z)-1$ of multiplicity $q$. We denote by $\bar{N}_{f \geq k+1}(r, 1 ; g \mid=m)$ the reduced counting functions of those 1-points of $f$ and $g$ for which $p \geq k+1$ and $q=m>0$.

Definition 1.10([8]). Let $a, b \in \mathbb{C} \cup\{\infty\}$. We denote by $N(r, a ; f \mid g \neq b)$ the counting function of those $a$-points of $f$, counted according to multiplicity, which are not the $b$-points of $g$.

Definition 1.11. Let $f$ and $g$ be two non-constant meromorphic functions such that $f$ and $g$ share the value 1 "IM". Let $z_{0}$ be a 1-point of $f$ with multiplicity $p$, a 1-point of $g$ with multiplicity $q$. We denote by $\bar{N}_{L}(r, 1 ; f)$ the counting function of those 1-points of $f$ and $g$ where $p>q$ and by $\bar{N}_{E}^{(2}(r, 1 ; f)$ the counting function of those 1-points of $f$ and $g$ where $p=q \geq 2$, each point in these counting functions is counted only once. In the same way we can define $\bar{N}_{L}(r, 1 ; g), \bar{N}_{E}^{(2}(r, 1 ; g)$.

\section{Lemmas}

In this section we present some lemmas which will be needed in the sequel. Let $F, G$ be two non-constant meromorphic functions. Henceforth we shall denote by 
$H$ the following function.

$$
H=\left(\frac{F^{\prime \prime}}{F^{\prime}}-\frac{2 F^{\prime}}{F-1}\right)-\left(\frac{G^{\prime \prime}}{G^{\prime}}-\frac{2 G^{\prime}}{G-1}\right) .
$$

Lemma 2.1([2]). If $F, G$ be share $(1,1)^{*}$ and $H \not \equiv 0$. Then

$$
\begin{aligned}
N^{E}(r, 1 ; F, G \mid \leq 1)= & \leq N(r, 0 ; H) \\
& \leq N(r, \infty ; H)+S(r, F)+S(r, G) .
\end{aligned}
$$

Lemma 2.2. If for two positive integers $p$ and $k, N_{p}(r, 0 ; L(f) \mid f \neq 0)$ denotes the counting function of those zeros of of $L(f)$ which are not the zeros of $f$, where a zero of $L(f)$ with multiplicity $m$ is counted $m$ times if $m \leq p$ and $p$ times if $m>p$ then

$$
\begin{aligned}
& N_{p}(r, 0 ; L(f) \mid f \neq 0) \\
\leq & N_{k}(r, 0 ; f)+k \bar{N}(r, \infty ; f)-\sum_{m=p+1}^{\infty} \bar{N}\left(r, 0 ; \frac{L(f)}{f} \mid \geq m\right)+S(r, f) .
\end{aligned}
$$

Proof. We omit the proof since the same can be carried out in the line of proof of Lemma $2.2[2]$.

Lemma 2.3([17]). For two positive integers $p$ and $k$

$$
N_{p}(r, 0 ; L(f)) \leq N_{p+k}(r, 0 ; f)+k \bar{N}(r, \infty ; f)+S(r, f) .
$$

Lemma 2.4([17]). For two positive integers $p$ and $k$

$$
N_{p}(r, 0 ; L(f)) \leq T(r, L(f))-T(r, f)+N_{p+k}(r, 0 ; f)+S(r, f) .
$$

Lemma 2.5. Let $f$, g share $(1,2)^{*}$. Then

$$
2 \bar{N}_{f \geq 3}(r, 1 ; g \mid=1)+\bar{N}_{f \geq 3}(r, 1 ; g \mid=2) \leq \bar{N}(r, 0 ; f)+\bar{N}(r, \infty ; f)+S(r) .
$$

Proof. Using Lemma 2.2 we get

$$
\begin{aligned}
2 \bar{N}_{f \geq 3}(r, 1 ; g \mid=1)+\bar{N}_{f \geq 3}(r, 1 ; g \mid=2) & =2 \bar{N}(r, 1 ; f \mid \geq 3)+S(r) \\
& \leq N_{2}\left(r, 0 ; f^{\prime} \mid f \neq 0\right)+S(r) \\
& \leq \bar{N}(r, 0 ; f)+\bar{N}(r, \infty ; f)+S(r) .
\end{aligned}
$$

Lemma 2.6([12]). Let $f$ be a non-constant meromorphic function and let

$$
R(f)=\frac{\sum_{k=0}^{n} a_{k} f^{k}}{\sum_{j=0}^{m} b_{j} f^{j}}
$$


be an irreducible rational function in $f$ with constant coefficients $\left\{a_{k}\right\}$ and $\left\{b_{j}\right\}$ where $a_{n} \neq 0$ and $b_{m} \neq 0$. Then

$$
T(r, R(f))=d T(r, f)+S(r, f)
$$

where $d=\max \{n, m\}$.

Lemma 2.7([5, p.68] $])$. Suppose that $f$ is meromorphic and transcendental in the plane and that

$$
f^{n} P=Q,
$$

where $P$ and $Q$ are differential polynomials in $f$ and the degree of $Q$ is at most $n$. Then

$$
m(r, P)=S(r, f) \text { as } r \longrightarrow+\infty .
$$

\section{Proofs of the theorems}

Proof of Theorem 1.3. Let $F=\frac{L(f)}{a}$ and $G=\frac{f}{a}$. Then $F-1=\frac{L(f)-a}{a}$ and $G-1=\frac{f-a}{a}$. Since $f-a$ and $L(f)-a$ share $(0,2)^{*}$ it follows that $F, G$ share $(1,2)^{*}$ except the zeros and poles of $a(z)$. Now we consider the following cases.

Case 1 Let $H \not \equiv 0$. Since $H$ has only simple poles, from (2.1) it can be easily calculated that

$$
\begin{aligned}
(3.1) N(r, \infty ; H) \leq & \bar{N}(r, \infty ; F)+\bar{N}_{L}(r, 1 ; F)+\bar{N}_{L}(r, 1 ; G)+\bar{N}(r, 0 ; F \mid \geq 2) \\
& +\bar{N}(r, 0 ; G \mid \geq 2)+\bar{N}_{0}\left(r, 0 ; F^{\prime}\right)+\bar{N}_{0}\left(r, 0 ; G^{\prime}\right) \\
& +\bar{N}(r, 0 ; a)+\bar{N}(r, \infty ; a),
\end{aligned}
$$

where $\bar{N}_{0}\left(r, 0 ; F^{\prime}\right)$ is the reduced counting function of those zeros of $F^{\prime}$ which are not the zeros of $F(F-1)$ and $\bar{N}_{0}\left(r, 0 ; G^{\prime}\right)$ is similarly defined. Now from Lemma 2.1 we have

$$
\begin{aligned}
(3.2) & \bar{N}(r, 1 ; F)+\bar{N}(r, 1 ; G) \\
\leq & N^{E}(r, 1 ; F, G \mid \leq 1)+\bar{N}_{E}^{(2}(r, 1 ; F)+\bar{N}_{L}(r, 1 ; F)+\bar{N}_{L}(r, 1 ; G) \\
& +\bar{N}(r, 1 ; G)+N(r, \infty ; a)+N(r, 0 ; a)+S(r, f) \\
\leq & N(r, \infty ; H)+\bar{N}_{E}^{(2}(r, 1 ; F)+\bar{N}_{L}(r, 1 ; F)+\bar{N}_{L}(r, 1 ; G)+\bar{N}(r, 1 ; G)+S(r, f) .
\end{aligned}
$$

By the second fundamental theorem, (3.1), (3.2) and noting that $\bar{N}(r, \infty ; F)=$ $\bar{N}(r, \infty ; G)+S(r, f)$ we get 


$$
\begin{aligned}
& T(r, F)+T(r, G) \\
\leq & \bar{N}(r, \infty ; F)+\bar{N}(r, 0 ; F)+\bar{N}(r, \infty ; G)+\bar{N}(r, 0 ; G) \\
& +\bar{N}(r, 1 ; F)+\bar{N}(r, 1 ; G)-N_{0}\left(r, 0 ; F^{\prime}\right)-N_{0}\left(r, 0 ; G^{\prime}\right) \\
& +S(r, F)+S(r, G) \\
\leq & 3 \bar{N}(r, \infty ; f)+N_{2}(r, 0 ; F)+N_{2}(r, 0 ; G) \\
& +\bar{N}_{E}^{(2}(r, 1 ; F)+2 \bar{N}_{L}(r, 1 ; F)+2 \bar{N}_{L}(r, 1 ; G) \\
& +\bar{N}(r, 1 ; G)+S(r, f) .
\end{aligned}
$$

Since $F$ and $G$ share $(1,2)^{*}$ we note that

$$
\begin{aligned}
& 2 \bar{N}_{L}(r, 1 ; F)+2 \bar{N}_{L}(r, 1 ; G)+\bar{N}_{E}^{(2}(r, 1 ; F)+\bar{N}(r, 1 ; G) \\
\leq & N_{3}(r, 1 ; G)+2 \bar{N}_{F \geq 3}(r, 1 ; G \mid=1)+\bar{N}_{F \geq 3}(r, 1 ; G \mid=2)+S(r, f) .
\end{aligned}
$$

Since $N_{2}(r, 0 ; F)=N_{2}(r, 0 ; L(f))+S(r, f)$, using (3.4) and Lemmas 2.3-2.5 we get from $(3.3)$

$$
\begin{aligned}
& T(r, L(f))+T(r, f) \\
\leq & 4 \bar{N}(r, \infty ; f)+N_{2}(r, 0 ; f)+N_{2}(r, 0 ; L(f))+\bar{N}(r, 0 ; L(f)) \\
& +N_{3}(r, a ; f)+S(r, f) \\
\leq & (4+k) \bar{N}(r, \infty ; f)+N_{2}(r, 0 ; f)+T(r, L(f))-T(r, f) \\
& +N_{2+k}(r, 0 ; f)+N_{1+k}(r, 0 ; f)+N_{3}(r, a ; f)+S(r, f) .
\end{aligned}
$$

So it follows that

$$
(4+k) \Theta(\infty ; f)+\delta_{2+k}(0 ; f)+\delta_{1+k}(0 ; f)+\delta_{2}(0 ; f)+\delta_{3}(a ; f) \leq 6+k,
$$

which contradicts (1.20).

Case 2 Let $H \equiv 0$. On integration we get from (2.1)

$$
\frac{1}{F-1} \equiv \frac{C}{G-1}+D,
$$

where $C, D$ are constants and $C \neq 0$. If there exist a pole $z_{0}$ of $f$ with multiplicity $p$ which is not a pole and zero of $a(z)$, then $z_{0}$ is the pole of $F$ with multiplicity $p+k$ and the pole of $G$ with multiplicity $p$. This contradicts (3.5). So using (3.5) we get

$$
N(r, \infty ; f) \leq N(r, 0 ; a)+N(r, \infty ; a)=S(r, f),
$$

and hence

$$
N(r, \infty ; L(f))=S(r, f) .
$$

So $\Theta(\infty ; f)=1$. Therefore the assertion (1.20) reduces to

$$
\delta_{2+k}(0 ; f)+\delta_{1+k}(0 ; f)+\delta_{2}(0 ; f)+\delta_{3}(a ; f)>2
$$


Suppose $D \neq 0$. From (3.5) we can deduce

$$
\frac{L(f)}{a}=\frac{(D+1) \frac{f}{a}+C-D-1}{D \frac{f}{a}+C-D}
$$

From (3.7) we have

$$
D f L(f)=a((D+1) f+a(C-D-1))-a(C-D) L(f)
$$

Hence using Lemma 2.7 we obtain from (3.8)

$$
T(r, L(f))=m(r, L(f))+N(r, \infty ; L(f))=S(r, f) .
$$

Using Lemma 2.6 from (3.5) we get $T(r, G)=T(r, F)+S(r, f)$. Since $a$ is a small function it follows from (3.7) that

$$
T(r, f)=T(r, L(f))+S(r, f)=S(r, f),
$$

which is absurd. Hence $D=0$ and so from (3.5) we get $G-1 \equiv C(F-1)$. If $C \neq 1$, then

$$
F \equiv \frac{G}{C}+1-\frac{1}{C}
$$

and $\bar{N}(r, 0 ; F)=\bar{N}(r, 1-C ; G)$. By the second fundamental theorem, Lemma 2.3 for $p=1$ and noting that $\bar{N}(r, \infty ; F)=S(r, f)$ we get

$$
\begin{aligned}
& 2 T(r, f) \\
\leq & 2 T(r, G)+S(r, f) \\
\leq & \bar{N}(r, \infty ; G)+\bar{N}(r, 0 ; G)+\bar{N}(r, 1 ; G)+\bar{N}(r, 1-C ; G)+S(r, f) \\
\leq & \bar{N}(r, 0 ; G)+\bar{N}(r, \infty ; f)+\bar{N}(r, 1 ; G)+\bar{N}(r, 0 ; F)+S(r, f) \\
\leq & \bar{N}(r, 0 ; f)+N_{1+k}(r, 0 ; f)+N_{3}(r, 1 ; G)+S(r, f) .
\end{aligned}
$$

Hence

$$
\Theta(0 ; f)+\delta_{1+k}(0 ; f)+\delta_{3}(a ; f) \leq 1 .
$$

So we have

$$
\begin{aligned}
& \delta_{2+k}(0 ; f)+\delta_{2}(0 ; f)+\delta_{1+k}(0 ; f)+\delta_{3}(a ; f) \\
\leq \quad & \delta_{2+k}(0 ; f)+\Theta(0 ; f)+\delta_{1+k}(0 ; f)+\delta_{3}(a ; f) \leq 2 .
\end{aligned}
$$

This contradicts (3.6). Hence $C=1$ and so $F \equiv G$, that is $f \equiv L(f)$. This completes the proof of the theorem.

Proof of theorem 1.2. Let $F$ and $G$ be defined as in Theorem 1.3. 
It follows that $F=L\left(\frac{f}{a}\right)$ and $G=\frac{f}{a}$ share " $(1, l)$ ". Clearly the counting function of the 1-points of $G$ with multiplicity greater than $k$ is $S(r, f)$. Since $l(\geq k)$ it follows that $F$ and $G$ practically share " $(1, \infty)$ ". Hence $\bar{N}_{L}(r, 1 ; F)+\bar{N}_{L}(r, 1 ; G)=S(r, f)$. Case 1 Let $H \not \equiv 0$. Since

$$
\begin{aligned}
& \bar{N}(r, 1 ; F)+\bar{N}(r, 1 ; G) \\
\leq & N^{E}(r, 1 ; F, G \mid \leq 1)+\bar{N}(r, 1 ; F \mid \geq 2)+\bar{N}(r, 1 ; G)+S(r, f) \\
\leq & \bar{N}(r, \infty ; H)+N_{2}(r, 1: G)+S(r, f),
\end{aligned}
$$

(3.3) changes to

$T(r, F)+T(r, G) \leq 3 \bar{N}(r, \infty ; f)+N_{2}(r, 0: F)+N_{2}(r, 0: G)+N_{2}(r, 1 ; G)+S(r, f)$,

which with the help of Lemmas 2.3-2.4 yields

$$
3 \Theta(\infty ; f)+\delta_{2+k}(0 ; f)+\delta_{2}(0 ; f)+\delta_{2}(a ; f) \leq 4 .
$$

The above expression contradicts (1.19).

Case 2 Let $H \equiv 0$. Integrating (2.1) we get (3.5). If $z_{0}$ be a pole of $f$ with multiplicity $p$, then $z_{0}$ is the pole of $F$ with multiplicity $p+k$ and the pole of $G$ with multiplicity $p$. This contradicts (3.5). It follows that $F$ and $G$ have no pole and so $F$ and $G$ are entire functions here. From (1.19) we know

$$
\delta_{2+k}(0 ; f)+\delta_{2}(0 ; f)+\delta_{2}(a ; f)>1 .
$$

Now using the same argument as used in the proof of Theorem 1.3 we can obtain from (3.5) that $D=0$. Again assuming $C \neq 1$ and following the same method in the proof of Theorem 1.3 we can obtain

$$
\Theta(0 ; f)+\delta_{1+k}(0 ; f)+\delta_{2}(a ; f) \leq 1
$$

which together with (3.10) leads to a contradiction. Hence $C=1$ and so $F \equiv G$, that is $f \equiv L(f)$. This completes the proof of the theorem.

Proof of theorem 1.1. Let $F$ and $G$ be defined as in Theorem 1.3.

It follows that $F-1$ and $G-1$ share " $(0, l)$ " and hence $F, G$ share " $(1, l)$ " except the zeros and poles of $a(z)$.

Case 1 Let $H \not \equiv 0$.

Subcase $1.1 l=2$. We first note that here (3.4) changes to

$$
\begin{aligned}
& 2 \bar{N}_{L}(r, 1 ; F)+2 \bar{N}_{L}(r, 1 ; G)+\bar{N}_{E}^{(2}(r, 1 ; F)+\bar{N}(r, 1 ; G) \\
\leq \quad & N_{3}(r, 1 ; G)+S(r, f) .
\end{aligned}
$$

So using (3.12), Lemmas 2.3-2.5 we get from (3.3) that

$$
3 \Theta(\infty ; f)+\delta_{2+k}(0 ; f)+\delta_{2}(0 ; f)+\delta_{3}(a ; f) \leq 4,
$$


which contradicts (1.13).

Subcase $1.2 l=1$. Here (3.4) changes to

$$
\begin{aligned}
& 2 \bar{N}_{L}(r, 1 ; F)+2 \bar{N}_{L}(r, 1 ; G)+\bar{N}_{E}^{(2}(r, 1 ; F)+\bar{N}(r, 1 ; G) \\
\leq & N_{3}(r, 1 ; G)+\bar{N}_{F \geq 3}(r, 1 ; G \mid=2)+S(r, f) .
\end{aligned}
$$

Using Lemma 2.2 we note that

$$
\begin{aligned}
\bar{N}_{F \geq 3}(r, 1 ; G \mid=2) & \leq \bar{N}(r, 1 ; F \mid \geq 3)+S(r, f) \\
& \leq \frac{1}{2} N_{2}\left(r, 0 ; F^{\prime} \mid F \neq 0\right)+S(r, f) \\
& \leq \frac{1}{2}(\bar{N}(r, 0 ; F)+\bar{N}(r, \infty ; F))+S(r, f) .
\end{aligned}
$$

So using (3.13), Lemmas 2.3-2.5 and the above inequality we again get from (3.3) that

$$
\frac{7+k}{2} \Theta(\infty ; f)+\delta_{2+k}(0 ; f)+\frac{1}{2} \delta_{1+k}(0 ; f)+\delta_{2}(0 ; f)+\delta_{3}(a ; f) \leq 5+\frac{k}{2},
$$

which contradicts (1.14).

Subcase $1.3 l=0$. So $(3.4)$ changes to

$$
\begin{aligned}
& 2 \bar{N}_{L}(r, 1 ; F)+2 \bar{N}_{L}(r, 1 ; G)+\bar{N}_{E}^{(2}(r, 1 ; F)+\bar{N}(r, 1 ; G) \\
\leq & N_{2}(r, 1 ; G)+2 \bar{N}_{L}(r, 1 ; F)+\bar{N}_{L}(r, 1 ; G)+S(r, f) .
\end{aligned}
$$

Also using Lemma 2.2 for $p=1$ we obtain

$$
\begin{aligned}
& 2 \bar{N}_{L}(r, 1 ; F)+\bar{N}_{L}(r, 1 ; G) \\
\leq & 2 \bar{N}(r, 1 ; F \mid \geq 2)+\bar{N}(r, 1 ; G \mid \geq 2)+S(r, f) \\
\leq & 2 \bar{N}\left(r, 1 ; F^{\prime} \mid F \neq 0\right)+\bar{N}\left(r, 1 ; G^{\prime} \mid G \neq 0\right)+S(r, f) \\
\leq & 2 \bar{N}(r, 0 ; F)+3 \bar{N}(r, \infty ; f)+\bar{N}(r, 0 ; G)+S(r, f) .
\end{aligned}
$$

Using (3.14), (3.15) and Lemmas 2.3-2.5 we get from (3.3)

$$
\begin{aligned}
T(r, L(f))+T(r, f) \leq & (6+2 k) \bar{N}(r, \infty ; f)+T(r, L(f))-T(r, f)+N_{2+k}(r, 0 ; f) \\
& +2 N_{1+k}(r, 0 ; f)+\bar{N}(r, 0 ; f)+N_{2}(r, 0 ; f) \\
& +N_{2}(r, a ; f)+S(r, f) .
\end{aligned}
$$

Hence it follows that

$(6+2 k) \Theta(\infty ; f)+\delta_{2+k}(0 ; f)+2 \delta_{1+k}(0 ; f)+\delta_{2}(0 ; f)+\Theta(0 ; f)+\delta_{2}(a ; f) \leq 2 k+10$ which contradicts (1.15).

Case 2 Let $H \equiv 0$. Integrating (2.1) we get (3.5). In this case also proceeding in the same way as done in the proof of Theorem 1.3 we can obtain

$$
\Theta(\infty ; f)=1 .
$$


So (1.13)-(1.15) respectively reduces to

$$
\begin{gathered}
\delta_{2+k}(0 ; f)+\delta_{2}(0 ; f)+\delta_{3}(a, f)>1, \\
\delta_{2+k}(0 ; f)+\frac{1}{2} \delta_{1+k}(0 ; f)+\delta_{2}(0 ; f)+\delta_{3}(a, f)>\frac{3}{2}
\end{gathered}
$$

and

$$
\delta_{2+k}(0 ; f)+2 \delta_{1+k}(0 ; f)+\delta_{2}(0 ; f)+\Theta(0 ; f)+\delta_{2}(a, f)>4 .
$$

Now again proceeding in the same way as done in Theorem 1.3, from (3.5) we can obtain $D=0$. Next supposing $C \neq 1$ and following the same procedure as done in the proof of Theorem 1.3 we can get (3.11), which together with (3.16)-(3.18) leads to contradictions. Hence $C=1$. So from (3.5) we have $f=L(f)$. This completes the proof of the theorem.

Acknowledgment. The author wish to thank the referee for his/her valuable comments and suggestions towards the improvement of the paper.

\section{References}

[1] A. Banerjee, Weighted sharing of a small function by a meromorphic function and its derivative, Comput. Math. Appl., 53(11)(2007), 1750-1761.

[2] A. Banerjee, Uniqueness of meromorphic functions sharing a small function with their derivatives, Math. Vesnik, 60(2008) 121-135.

[3] A. Banerjee, and S. Mukherjee, Uniqueness of meromorphic functions concerning differential monomials sharing the same value, Bull. Math. Soc. Sci. Math. (Tome), 50(3) (2007), 191-206.

[4] R. Brück, On entire functions which share one value CM with their first derivative, Results Math., 30(1996), 21-24.

[5] W. K. Hayman, Meromorphic Functions, The Clarendon Press, Oxford, 1964.

[6] I. Lahiri, Weighted sharing and uniqueness of meromorphic functions, Nagoya Math. J., 161(2001), 193-206.

[7] I. Lahiri, Weighted value sharing and uniqueness of meromorphic functions, Complex Var. Theory Appl., 46(2001), 241-253.

[8] I. Lahiri, and A. Banerjee, Weighted sharing of two sets, Kyungpook Math. J. 46(1)(2006), 79-87.

[9] I. Lahiri, and A. Sarkar, Uniqueness of meromorphic function and its derivative, J. Inequal. Pure Appl. Math., 5(1)(2004), Art.20 [ONLINE http://jipam.vu.edu.au/].

[10] S. Lin and W. C. Lin, Uniqueness of meromorphic functions concerning weakly weighted sharing, Kodai Math. J., 29(2006), 269-280. 
[11] L. Liu and Y. Gu, Uniqueness of meromorphic functions that share one small function with their derivatives, Kodai Math. J., 27(2004), 272-279.

[12] A. Z. Mohon'ko, On the Nevanlinna characteristics of some meromorphic functions, Theory of Functions. Functional Analysis and Their Applications, 14(1971), 83-87.

[13] H. X. Yi, On characteristic function of a meromorphic function and its derivative, Indian J. Math., 33(2)(1991), 119-133.

[14] K. W. Yu, On entire and meromorphic functions that share small functions with their derivatives, J. Inequal. Pure Appl. Math., 4(1)(2003), Art.21 [ONLINE http://jipam.vu.edu.au/].

[15] Q.C.Zhang, Meromorphic function that shares one small function with its derivative, J. Inequal. Pure Appl. Math., 6(4)(2005), Art.116 [ ONLINE http://jipam.vu.edu.au/].

[16] J. L. Zhang and L. Z. Yang, Some results related to a conjecture of R.Brück concerning meromorphic functions sharing one small function with their derivatives, Ann. Acad. Sci. Fenn. Math., 32(2007), 141-149.

[17] J. L. Zhang and L. Z. Yang, Some results related to a conjecture of R.Brück, J. Inequal. Pure Appl. Math., 8(1)(2007), Art.18, 11pp. [ ONLINE http://jipam.vu.edu.au/]. 